\title{
Medroxyprogesterone acetate [Depo Provera] injections. Development of Striae
}

\author{
Mamta Gupta, MBBS, MFFP, BASRT Accredited \\ Senior Clinical Medical Officer, Family Planning and Reproductive Health, Bedfordshire and Luton Community NHS Trust, \\ Luton, $U K$
}

(Accepted December 6th 1999)

\begin{abstract}
Summary
A young girl developed extensive striae while on Depo Provera injections. She had gained some weight during the treatment. Endocrinal investigations were negative. The development of striae was attributed to her weight gain.
\end{abstract}

\section{Key words}

abdomen, buttocks, legs, medroxyprogesterone acetate, striae, thighs, upper arms, weight gain

\section{Key message points \\ - Contraceptive injection of medroxyprogesterone acetate led to the development of striae (stretch marks) in a young girl. \\ - Striae developed following the first injection, with a total weight gain of only $3.1 \mathrm{~kg}$. \\ - The striae were florid and distributed on her abdomen, upper arms, thighs, buttocks, back and legs. \\ - Pharmacia and Upjohn's database shows that for most cases no opinions were provided as to the cause, and the outcome of events were not reported or were not known.}

\section{Case report}

A twenty-year-old girl attended the family planning clinic requesting Depo Provera injection because she was feeling nauseated while on the combined oral contraceptive pill. She also complained that she forgot to take pills regularly, thereby making contraception inefficient. After a full discussion of its side effects and mode of action, she was given her first injection of $150 \mathrm{mg}$ Depo Provera by deep intramuscular route. Her weight on this visit was $74.2 \mathrm{~kg}$.

She returned 12 weeks later for her second injection. She had put on $3.1 \mathrm{~kg}$ in weight $(77.3 \mathrm{Kg})$. She reported stretch marks on her lower abdomen and upper arms as well as a few mood swings. She commented that she had recently returned from a holiday where she had eaten a lot of chips and other fatty foods. At this point the stretch marks were attributed to the weight gain and dietary advice was given.

She was offered the combined pill once again as an alternative but declined it because she said that she was very forgetful and would prefer to continue with the injections. She was not interested in any other method of contraception. She received her second dose of Depo Provera at this visit. She did not contact the clinic until her third injection was due, when her weight had gone up by a further $2.6 \mathrm{~kg}(79.9 \mathrm{Kg})$. Her striae had now become quite florid and were distributed on her abdomen, upper arms, thighs, buttocks, back and legs (Figure 1). No other symptoms were reported. She was understandably very upset. Following discussion, she decided to switch to progesterone only pill, her boyfriend promising to ensure that she took the pills on time. She did not want to go back on the combined oral contraceptive pill or try any of the other non-hormonal methods.

On her next visit the following month, she was continuing to gain weight. It is not definite that she followed any dietary advice. She did not feel that her weight gain had anything to do with her eating habits. All the information, including Medline search results, were discussed with her at each stage. At this point, she was referred to the consultant endocrinologist to exclude endocrine problems. She was found to have a normal cortisol level and had a normal overnight dexamethazone suppression test. The consultant's view was that her overall weight gain of 5-6 Kg at a height of $154 \mathrm{~cm}$ was probably sufficient to cause the striae. This was explained to her, and the importance of controlling her weight was stressed.

On her subsequent visits, she continued to gain weight. Dietary advice was not followed. Her striae became less florid as the time progressed, but still continued to cause her distress.

Figure 1 Depo Provera injections and striae. Injections were given by deep intramuscular route in the gluteal muscle, alternating sides.
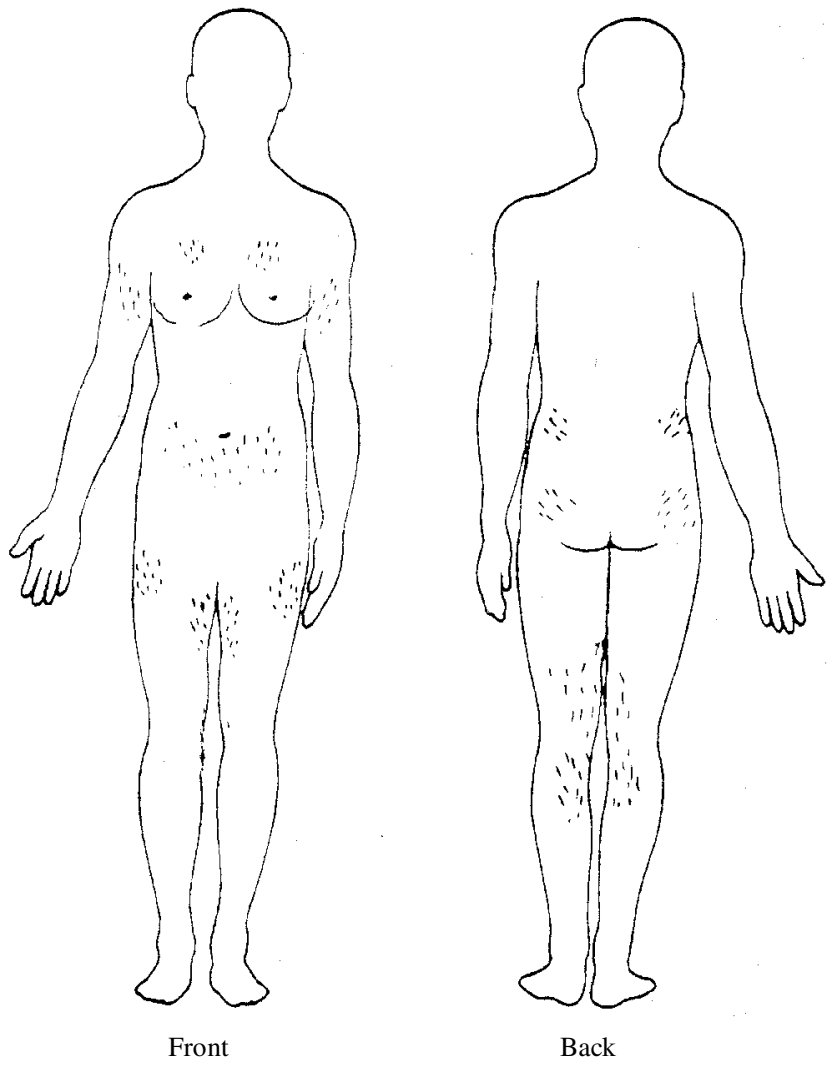
A search through Pharmacia and Upjohn, the manufacturer, showed very few reported cases of striae developing following Depo Provera injection. The most recent search of their world-wide spontaneous adverse events database (completed $28^{\text {th }}$ April 98) shows that they have received 28 reports of striae in women on Depo Provera since market introduction of the product in 1960. Where known, onset of symptoms ranged from 2 days to 4 years following the first Depo Provera injection. A full recovery was reported in only one case. In this case, the striae appeared 6 months after starting the Depo Provera and the event resolved spontaneously without treatment about 4 months later while the patient continued Depo Provera. In most cases the outcome of the events was not reported or was unknown.

In the reports received by the company, no opinions were provided as to the cause of the striae. In 10 reports, however, weight gain and/or a history of weight problems were noted. One of the cases from UK was published where authors suggested that the striae might have been the result of an idiosyncratic reaction to Depo Provera. ${ }^{1}$ No other published references were found.

The data from Pharmacia and Upjohn are from a spontaneous medical event reporting system, which consists of a world-wide database of unsolicited reports. As with all spontaneous reporting systems, there is not necessarily a causal relationship between the use of a drug and the occurrence of an event. The numbers of events collected through this system must be interpreted as reporting rates and not incidence rates. ${ }^{2}$

Statements on funding and competing interests

Funding. None.

Competing interests. None declared.

References

Harris D, Ridley CM. Striae from Depo Provera injections [Letter]. Clin Exp Dermatol 1996;

2 Pharmacia and Upjohn Database. Data on file, April 1998 